gerschaftliches Engagement kein Gegenstand von „Sonntagsreden“ bleiben muss, sondern auch in direkter Konfrontation mit der „offiziellen Gemeindepolitik“ ein diskutierfähiger Gegenstand ist, dem es mit Respekt zu begegnen gilt.

Ein harmonischer Abschluss der Befragung wird durch die Frage nach der Praxisbewährung erzielt; sie verdeutlicht, dass direkte Demokratie mit der Repräsentativstruktur der Kommunalpolitik verträglich ist, weil der punktuelle Eingriff keine Außerkraftsetzung eines allgemein bewährten Systems zur Folge hat: „Die konzeptionelle Gesamtverantwortung und die Wahrung der Kontinuität kommunaler Politik liegt nach wie vor beim Rat. "30 Die meisten Kommentare der Gemeinden weisen in die Richtung einer sukzessiv wachsenden Toleranz gegenüber direktdemokratischen Handlungsoptionen ${ }^{31}$. Für künftige empirische Befragungen empfiehlt es sich im Übrigen generell, neben dem starren Ankreuzen vorformulierter Antwortalternativen eine kommentierende Begleitung zuzulassen und die gesammelten Einzelmeinungen so zu verdichten, dass sich daraus Argumentationslinien offenbaren können.

30 Fritz Ossenbühl, a.a.O., S. 253.

31 Die Ausgangsfrage ist insoweit dahingehend zu beantworten, dass eine legitime und auch gewollte Bereicherung der Lokalpolitik stattfindet.

\title{
Köpfe statt Themen? Köpfe und Themen! Die Personalisierung der Wahlkampfberichterstattung in Deutschland und in den USA*
}

\author{
Kim Jucknat
}

Die Personalisierung der Politik ist nicht erst seit heute ein zentrales Thema der Wahlkampfkommunikationsforschung ${ }^{1}$. Der frühere Wahlkampfmanager der CDU Peter Radunski bemerkte schon Anfang der achtziger Jahre: „Politische Führer als Verkörperung politischer Ideale hat es zu allen Zeiten gegeben." 2 Betrachtet man den Wahlwerbeslogan der CDU beim Bundestagswahlkampf 1969 „Auf den Kanzler kommt es an“ oder etwa

* Der Beitrag basiert auf der unveröffentlichten Magisterarbeit aus dem Jahr 2005 mit dem Titel „Politikvermittlung in parlamentarischen und präsidentiellen Demokratien“, Universität Mannheim, Lehrstuhl für Politische Wissenschaft und International Vergleichende Sozialforschung Prof. Dr. Jan van Deth; Datensatz und Manuskript können auf Anfrage eingesehen werden.

1 Vgl. etwa Helmut Norpoth, Kanzlerkandidaten, in: PVS, 18. Jg. (1977), S. 551 - 572; Max Kaase, Is there personalization in politics? Candidates and Voting Behaviour in Germany, in: International Political Science Review, 15. Jg. (1994), S. 211 - 230; Peter Radunski, Wahlkämpfe. Moderne Wahlkampfführung als politische Kommunikation, München 1980; Klaus Schönbach / Holli Semetko, Medienberichterstattung und Parteienwerbung im Bundestagswahlkampf 1990, in: Media Perspektiven, 7. Jg. (1994), S. 328 - 340.

2 Peter Radunski, a.a.O., S. 15. 
den Wahlwerbeslogan der SPD beim Bundestagswahlkampf 2005 „Gerhard Schröder muss Kanzler bleiben“, hat es den Anschein, dass sich die Personalisierung der Politik in Deutschland mittlerweile als fester Bestandteil von Wahlkämpfen etabliert hat. „Amerikanisierung" lautet das Stichwort in diesem Zusammenhang: Deutsche Wahlkämpfe - so scheint es - nähern sich immer mehr den professionalisierten US-amerikanischen $\mathrm{an}^{3}$, die sich durch hochgradige Personalisierung auszeichnen. Diese wird vorrangig mit Dethematisierung und Entideologisierung in Verbindung gebracht ${ }^{4}$. „Images“ statt „issues “5 lautet der Vorwurf, politische Sachfragen und politisch-ideologische Auseinandersetzungen würden von negative campaigning, horse race-journalism ${ }^{6}$, Privatisierung und der Thematisierung des Wahlkampfes per se verdrängt.

Hier wird die Personalisierung der Politik jedoch aus einer Perspektive betrachtet, die Personen und Themen als Einheit versteht, denn Personalisierung ist ein wirksames und notwendiges Mittel, um politische Themen zu vermitteln: Sie wirkt für den Wähler komplexitätsreduzierend, und die Darstellung von Themen in Verbindung mit Personen ist nachhaltiger ${ }^{7}$. Die oben angesprochenen Konvergenzen bei deutschen Wahlkämpfen im Vergleich zu US-amerikanischen sollen im Folgenden mittels einer quantitativen Inhaltsanalyse der Wahlkampfberichterstattung führender Printmedien in Deutschland und den USA beantwortet werden. Die Leitfragen der empirischen Analyse lauten dabei: Nähert sich die deutsche Wahlkampfberichterstattung der US-amerikanischen an, beziehungsweise wie ist die Entwicklung der Personalisierung der deutschen Wahlkampfberichterstattung im Vergleich zur US-amerikanischen zu bewerten? Inwiefern schlagen die unterschiedlichen institutionellen Rahmenbedingungen der beiden Länder zu Buche?

3 Vgl. Fritz Plasser, Globalisierung der Wahlkämpfe. Praktiken der Campaign Professionals im weltweiten Vergleich, Wien 2003; Patrick Donges, Amerikanisierung, Professionalisierung, Modernisierung? Anmerkungen zu einigen amorphen Begriffen, in: Klaus Kamps (Hrsg.), Trans Atlantik - Trans - Portabel? Die Amerikanisierungsthese in der politischen Kommunikation, Wiesbaden 2000, S. 27 - 42; Christina Holtz-Bacha, Wahlkampf in Deutschland. Ein Fall bedingter Amerikanisierung, in: ebenda, S. 43 - 56.

4 Vgl. etwa Fritz Plasser, a.a.O., S. 106; Robert E. Denton / Gary C. Woodward, Political Communication in America, 2. Auflage, New York / Westport, Connecticut / London 1990, S. 106.

5 Christina Holtz-Bacha, Massenmedien und Wahlen: Die Professionalisierung der Kampagnen, in: APuZ, B 15-16 (2002), S. $23-28$.

6 Das Konzept des „horse race journalism“ beschreibt mediale Wahlkampfberichterstattung, die den inhaltlichen Fokus auf die konkurrierenden Kandidaten legt und deren Wettstreit als so genanntes horse race (Pferderennen) spannend aufbereitet. „Negative campaigning“ ist ebenfalls eine mediale Darstellungsstrategie. Sie zielt darauf ab, publikumswirksame Skandale und Kontroversen in Bezug auf einen Kandidaten zu berichten.

7 Vgl. Robert E. Denton / Gary C. Woodward, a.a.O, S. 158; Peter Radunski, Politisches Kommunikationsmanagement. Die Amerikanisierung der Wahlkämpfe, in: Bertelsmann Stiftung (Hrsg.), Politik überzeugend vermitteln. Wahlkampfstrategien in Deutschland und den USA. Analysen und Bewertungen von Experten, Gütersloh 1996, S. 33 - 52, S. 40; Volker Hetterich, Von Adenauer bis Schröder - der Kampf um Stimmen. Eine Längsschnittanalyse der Wahlkampagnen von CDU und SPD bei den Bundestagswahlen 1949-1998, Opladen 2000, S. 56. 


\section{Die Rolle der Medien im Wahlkampf}

Repräsentative Demokratien basieren auf dem Prinzip der Vertretung politischer Interessen der Bürger durch Entscheidungsträger. Durch Wahlen kann der Bürger seine politischen Interessen artikulieren und zum Ausdruck bringen. Da der regelmäßige persönliche Kontakt des Wählers mit dem Gewählten nicht möglich ist, bedarf es für die politische Kommunikation eines Vermittlers. Diese Funktion nehmen die Massenmedien in modernen Demokratien ein ${ }^{8}$. Über sie steht der Bürger in Verbindung mit den Politikern; die Medien sind es, die den Informationsfluss zwischen Regierenden und Regierten sicherstellen: Moderne Demokratien sind „Mediengesellschaften“, die sich durch eine zentrale Rolle des Mediensektors auszeichnen?.

$\mathrm{Zu}$ Wahlkampfzeiten wird diese Rolle besonders deutlich. Studien ${ }^{10}$ belegen, dass es neben der direkten persönlichen Kommunikation die „von den Medien vermittelten Überzeugungsbotschaften " sind ${ }^{11}$, welche die Wahlentscheidung der Bürger in erheblichem Maße beeinflussen. Die Medien gewährleisten die Vermittlung der Wahlkampfbotschaften der politischen Parteien an die Wähler und ermöglichen gleichzeitig eine Rückkoppelung. Alle drei Akteure - Medien, Parteien und Wähler - beeinflussen sich gegenseitig $^{12}$, so dass sich Veränderungen bei einem rekursiv auf die anderen auswirken. Da sich heutzutage ohne die Massenmedien kein Wahlkampf mehr gewinnen lässt, müssen sich die Parteien weitgehend der Medienlogik anpassen, also ihre Wahlkampfstrategien auf die Strukturen und Arbeitsweisen der Medien ausrichten ${ }^{13}$. Als „Medienkommunikationsparteien"14, die ihre politischen Botschaften mittels professionalisierter Kommunikationsstrategien wie etwa der Inszenierung von Pseudoereignissen und der Personalisierung von Themen auf der Medienagenda platzieren, kann es den Parteien gelingen, die Wähler zu erreichen. Daraus folgt, dass die Analyse medialer Berichterstattung Einblicke in die Ausgestaltung und Entwicklung von Wahlkampfstrategien erlaubt.

\section{Datenbasis und Datenerhebung}

Für die hier vorzunehmende Analyse wurde die Berichterstattung über fünf Bundestagswahlkämpfe in Deutschland und fünf Präsidentschaftswahlkämpfe in den USA unter-

8 Vgl. Robert E. Denton / Gary C. Woodward, a.a.O, S. 144; Katrin Voltmer, Medienqualität und Demokratie. Eine empirische Analyse publizistischer Informations- und Orientierungsleistungen in der Wahlkampfkommunikation, Baden-Baden 1998, S. 13.

9 Vgl. Ulrich Saxer, System, Systemwandel und politische Kommunikation, in: Otfried Jarren I Ulrich Sarcinelli / Ulrich Saxer (Hrsg.), Politische Kommunikation in der demokratischen Gesellschaft. Ein Handbuch mit Lexikonteil, Opladen / Wiesbaden 1998, S. 21 - 64, S. 46.

10 Rüdiger Schmitt-Beck, Politische Kommunikation und Wählerverhalten. Ein internationaler Vergleich, Wiesbaden 2002.

11 Ebenda, S. 96.

12 Vgl. Hans-Dieter Klingemann / Katrin Voltmer, Politische Kommunikation als Wahlkampfkommunikation, in: Otfried Jarren / Ulrich Sarcinelli / Ulrich Saxer (Hrsg.), a.a.O., S. 396 - 405.

13 Vgl. Uwe Jun, Der Wandel von Parteien in der Mediendemokratie. SPD und Labour Party im Vergleich, Frankfurt am Main 2004.

14 Ebenda, S. 125. 
sucht. Einbezogen wurden für Deutschland die Bundestagswahlkämpfe der Jahre 1953, 1961, 1972, 1987 und 2002, für die USA die Präsidentschaftswahlkämpfe der Jahre 1956, 1960, 1972, 1988 und $2000^{15}$.

Es wurden jeweils zwei überregionale Tageszeitungen in Deutschland und den USA ausgewertet: die „Frankfurter Allgemeine Zeitung“ (FAZ) und die „Süddeutsche Zeitung" (SZ) für Deutschland sowie die „New York Times" (NYT) und die „Washington Post“ (WP) ${ }^{16}$ für die USA. Die SZ und die FAZ zählen zu den auflagenstärksten Zeitungen Deutschlands; überdies wird ihnen das Prädikat der „Leitmedien“ zugesprochen ${ }^{17}$. Die NYT und die WP zählen in den USA ebenfalls zu den Tageszeitungen mit nationaler Verbreitung und haben Leitmediencharakter. Ferner gehören sie zu den fünf auflagenstärksten Zeitungen des Landes; ihre Berichterstattung erreicht wie bei den ausgewählten deutschen Zeitungen einen großen Bevölkerungsanteil ${ }^{18}$.

Bei allen ausgewählten Medien wurden die Artikel der Titelseite analysiert. Es wurden jene in die Auswertung einbezogen, die sich ausschließlich auf die Vermittlung von politischen Informationen beschränkten. Artikel, die eindeutig als Kommentare von Journalisten identifiziert werden konnten, Meinungen und Wertungen enthielten, wurden nicht berücksichtigt. Die Berichterstattung wurde in der "heißen Phase“ des Wahlkampfes untersucht, die den Zeitraum von vier Wochen vor dem Wahltermin umfasst ${ }^{19}$. In dieser letzten Phase verstärken sich die Anstrengungen der zur Wahl stehenden politischen Akteure noch einmal ${ }^{20}$, die Politikvermittlung ist hier am intensivsten. Die Stichprobe umfasste jeden dritten Kalendertag; so war die tagesaktuelle und längsschnittliche Breite des Datenmaterials nicht beeinträchtigt: Für jeden untersuchten Wahlkampf wurden somit zehn Titelseiten pro ausgewählter Zeitung analysiert.

Da die Codierung von der Autorin selbst vorgenommen wurde, stellte sich das Problem der Intercoderreliabilität nicht. Diese wurde dadurch gewährleistet, dass die Autorin während des laufenden Codiervorgangs Zufallsstichproben bereits codierter Dokumente mit der Größe von jeweils zwei Analyseeinheiten je Medium und je analysiertem Wahlkampf ein zweites Mal codiert und die Ergebnisse überprüft hat: Dabei konnten in keinem der Fälle gravierende Abweichungen festgestellt werden.

Die Codiereinheit bildete der Satz beziehungsweise Quasi-Satz. Das für den Codiervorgang verwendete Kategorienschema setzt sich aus zwei Analyseebenen zusammen: „Politische Akteure“ und „Themen“ (vgl. Tabelle 1). Mit dem Kategorienschema für die

15 Da die empirische Analyse Aufschlüsse über die Entwicklung der Wahlkampfberichterstattung beider Länder geben soll, wurde darauf geachtet, dass die ausgewählten Wahlkämpfe jeweils das gesamte Spektrum der Typologie der Wahlkampfphasen abdecken (vormodern, modern, professionalisiert); zur Typologie der Wahlkampfphasen vgl. Pippa Norris, A Virtuous Circle. Political Communications in Postindustrial Societies, Cambridge 2000.

16 Im Folgenden werden die Zeitungen mit den oben in Klammern gesetzten Abkürzungen benannt.

17 Vgl. Elisabeth Noelle-Neumann / Winfried Schulz / Jürgen Wilke (Hrsg.), Fischer Lexikon Massenkommunikation, 5. erweiterte Auflage, Frankfurt am Main 1999, S. 393 f.

18 Vgl. Hans J. Kleinsteuber, Medien und öffentliche Meinung, in: Willi Paul Adams / Peter Lösche (Hrsg.), Länderbericht USA, Bonn 1998, S. 375 - 392, S. 377.

19 Vgl. Jürgen Wilke / Carsten Reinemann, Kanzlerkandidaten in der Medienberichterstattung. Eine vergleichende Studie zu den Bundestagswahlen 1949-1998, Köln u. a. 2000, S. 21.

$20 \mathrm{Vgl}$. Klaus Kindelmann, Kanzlerkandidaten in den Medien. Eine Analyse des Wahljahres 1990, Opladen 1994, S. 49. 


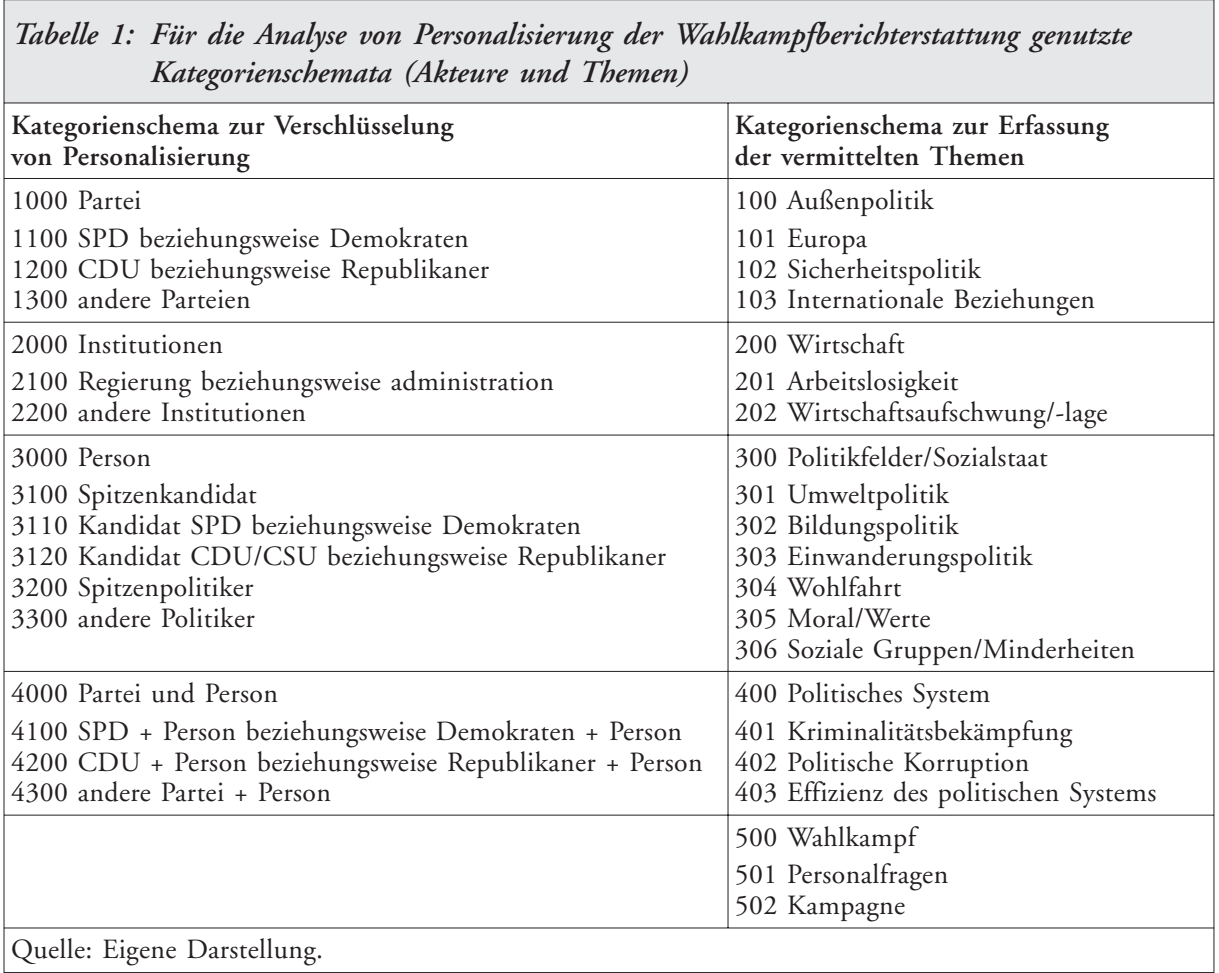

Analyseebene „Politische Akteure“ soll herausgefunden werden, welche Akteure bei der Personalisierung im Vordergrund stehen. Das Kategorienschema für die Analyseebene "Themen“ zeigt die Themen auf, die in Verbindung mit den politischen Akteuren vermittelt werden.

Das Kategorienschema „Politische Akteure“ setzt sich aus vier Dimensionen zusammen: „Partei“, „Institutionen“, „Person“ und „Partei und Person“. Unter der Dimension „Partei“ wurden jeweils die zwei großen Parteien (D: CDU/CSU und SPD; USA: Republikaner und Demokraten) gefasst. Unter „Institutionen“ fielen die Regierung in Deutschland als Exekutive und die „Administration“ in den USA als exekutive Kraft. Unter „Person“ wurden jeweils die Spitzenkandidaten der beiden großen Parteien vercodet (D: Kanzlerkandidat CDU/CSU und SPD; USA: Spitzenkandidat Republikaner und Demokraten), also diejenigen Politiker, die von den großen Parteien als Kanzlerkandidat beziehungsweise Präsidentschaftskandidat im Wahlkampf präsentiert wurden. „Spitzenpolitiker“"wurden für beide Länder als die Gruppe definiert, die nationalen Einfluss besitzt. In den USA gehören dazu unter anderem Parteivorsitzende, Mitglieder der „Administration", Gouverneure, Regierungssprecher und Kongressabgeordnete; in Deutschland fallen in diese Kategorie Fraktionsvorsitzende, Parteivorsitzende, Regierungssprecher und Minister. ${ }^{21}$ Mit der Dimension „Partei und Person“ wurden Nennungen von Politikern

21 Regierungssprecher wurden in die Kategorie „Spitzenpolitiker“ aufgenommen, da ihnen aufgrund ihrer repräsentativen Funktion vergleichbare mediale Aufmerksamkeit zuteil wird wie profilierten Politikern mit nationalem Einfluss. 
Tabelle 2: Datenbasis (Dokumente und Fälle) für den Vergleich der Wablkampfberichterstattung in Deutschland und den USA

\begin{tabular}{|c|c|c|c|c|c|c|c|c|}
\hline & \multicolumn{4}{|c|}{ USA } & \multicolumn{4}{|c|}{ Deutschland } \\
\hline & \multicolumn{4}{|c|}{ Analyseeinheiten } & \multicolumn{4}{|c|}{ Analyseeinheiten } \\
\hline & \multicolumn{2}{|c|}{ WP } & \multicolumn{2}{|c|}{ NYT } & \multicolumn{2}{|c|}{ FAZ } & \multicolumn{2}{|c|}{$S Z$} \\
\hline Wahlkampfjahr & $\begin{array}{l}\text { Analyse- } \\
\text { einheiten }\end{array}$ & $\begin{array}{l}\text { Codier- } \\
\text { einheiten }\end{array}$ & $\begin{array}{l}\text { Analyse- } \\
\text { einheiten }\end{array}$ & $\begin{array}{l}\text { Codier- } \\
\text { einheiten }\end{array}$ & $\begin{array}{l}\text { Analyse- } \\
\text { einheiten }\end{array}$ & $\begin{array}{l}\text { Codier- } \\
\text { einheiten }\end{array}$ & $\begin{array}{l}\text { Analyse- } \\
\text { einheiten }\end{array}$ & $\begin{array}{l}\text { Codier- } \\
\text { einheiten }\end{array}$ \\
\hline $1950 \mathrm{er}$ & 10 & 1217 & 10 & 1563 & 10 & 911 & 10 & 873 \\
\hline $1960 \mathrm{er}$ & 10 & 1186 & 10 & 1495 & 10 & 1034 & 10 & 906 \\
\hline $1970 \mathrm{er}$ & 10 & 889 & 10 & 977 & 10 & 993 & 10 & 973 \\
\hline $1980 \mathrm{er}$ & 10 & 913 & 10 & 894 & 10 & 1121 & 10 & 924 \\
\hline \multirow[t]{2}{*}{$2000 \mathrm{er}$} & 10 & 711 & 10 & 931 & 10 & 944 & 10 & 821 \\
\hline & 50 & 4916 & 50 & 5860 & 50 & 5003 & 50 & 4497 \\
\hline
\end{tabular}

zusammen mit ihrer Partei erfasst: Hier wurden jeweils wieder nur die beiden großen Parteien in beiden Ländern berücksichtigt.

Das Kategorienschema zur Erfassung der Themen orientiert sich am „Manifesto Research Coding Scheme", das zur Analyse von Parteiprogrammen entwickelt wurde. Dieses Schema diente als Richtlinie zur Entwicklung der aufgeführten Kategorien, da ein Pretest ergab, dass es für die Analyse der politischen Inhalte der Zeitungen zu ausdifferenziert war. Wie das Manifesto Research Group (MRG)-Schema beruht das hier verwendete Kategorienschema auf „salienztheoretischen“ Annahmen, nach denen politische Parteien miteinander konkurrieren, indem sie die einzelnen Themen aus der identischen Palette an politischen Themen anders gewichten, ihnen jeweils eine andere Bedeutung, eine andere "Salienz" zusprechen ${ }^{22}$.

Jeder Satz wurde also zweimal vercodet, mit einem Code für die Analyse der Personalisierung und einem Code für die Analyse des vermittelten Themas. Da sich die Fragestellung auf die Personalisierung in Verbindung mit der Vermittlung politischer Themen bezieht, wurden diejenigen Einheiten mit „0“ codiert, die nicht mit dem Kategorienschema der Analyseebene „Personalisierung" erfasst werden konnten: Wenn in einem Satz kein politischer Akteur genannt wurde, das heißt, wenn ein Thema in Verbindung mit einem Akteur vermittelt wurde, der nicht im Kategorienschema der Analyseebene „Politische Akteure“ aufgeführt war, wurde dieser Satz nur einmal mit „0“ erfasst.

So konnten beispielsweise für die folgende Codiereinheit zwei Codes vergeben werden: „Außenminister Scheel dankte der jugoslawischen Regierung für ihr promptes und kooperatives Verhalten.“ Außenminister Scheel wurde mit der Analyseebene „Politische Akteure" erfasst und als „Spitzenpolitiker" codiert. Auf der Analyseebene „Themen" wurde der Kode "Sicherheitspolitik" vergeben, da in diesem Satz die Zusammenarbeit Deutschlands mit Jugoslawien zur Bekämpfung der Flugzeugentführungen genannt wurde. ${ }^{23}$ Ein Beispiel für eine mit "0“ erfasste Kodiereinheit stellt der folgende Satz dar:

22 Ian Budge / Dennis Farlie, Party Competition - Selective Emphasis or Direct Confrontation? An Alternative View with Data, in: Hans Daalder / Peter Mair (Hrsg.), Western European Party Systems. Continuity \& Change, London 1983, S. 267 - 305.

23 Frankfurter Allgemeine Zeitung vom 31. Oktober 1972, Artikel über Erhöhung der Sicherheitsmaßnahmen zum Schutz vor Flugzeugentführungen. 
„Nach dem zweiten TV-Duell begannen selbst professionelle Beobachter an der Veranstaltung zu zweifeln. “24 Diese Codiereinheit beinhaltet keinen der im Kategorienschema aufgeführten Akteure und wurde daher mit „0“ erfasst. Für den Codiervorgang ergaben sich, wie aus Tabelle 2 hervorgeht, insgesamt 200 Analyseeinheiten und 20.276 Kodiereinheiten.

\section{Empirische Befunde}

\subsection{Vergleich Deutschland - USA}

Der Anteil an Themen, der in Deutschland in Verbindung mit politischen Akteuren vermittelt wurde, ist bis auf die fünfziger Jahre immer größer als in den USA (vgl. Abbildung 1). Für die USA kann konstatiert werden, dass die Bedeutung politischer Akteure in der Wahlkampfberichterstattung nicht zugenommen hat. In Deutschland kann man ebenfalls von keinem klaren Anstiegstrend sprechen. Es ist allerdings auffällig, in welchem Verhältnis die beiden Entwicklungen zueinander stehen. Seit den sechziger Jahren werden in Deutschland im Vergleich zu den USA mehr Themen in Verbindung mit politischen Akteuren vermittelt. Das ist ein überraschendes Ergebnis, wäre doch für die USA, als Land, in dem die Wahlkämpfe den höchsten Professionalisierungsgrad aufweisen, eine stärkere Konzentration auf politische Akteure in der Wahlkampfberichterstattung zu erwarten gewesen. Neben den Kanzlerkandidaten kommt in Deutschland noch einigen anderen politischen Akteuren im Wahlkampf Bedeutung zu, und diese Tatsache spiegelt

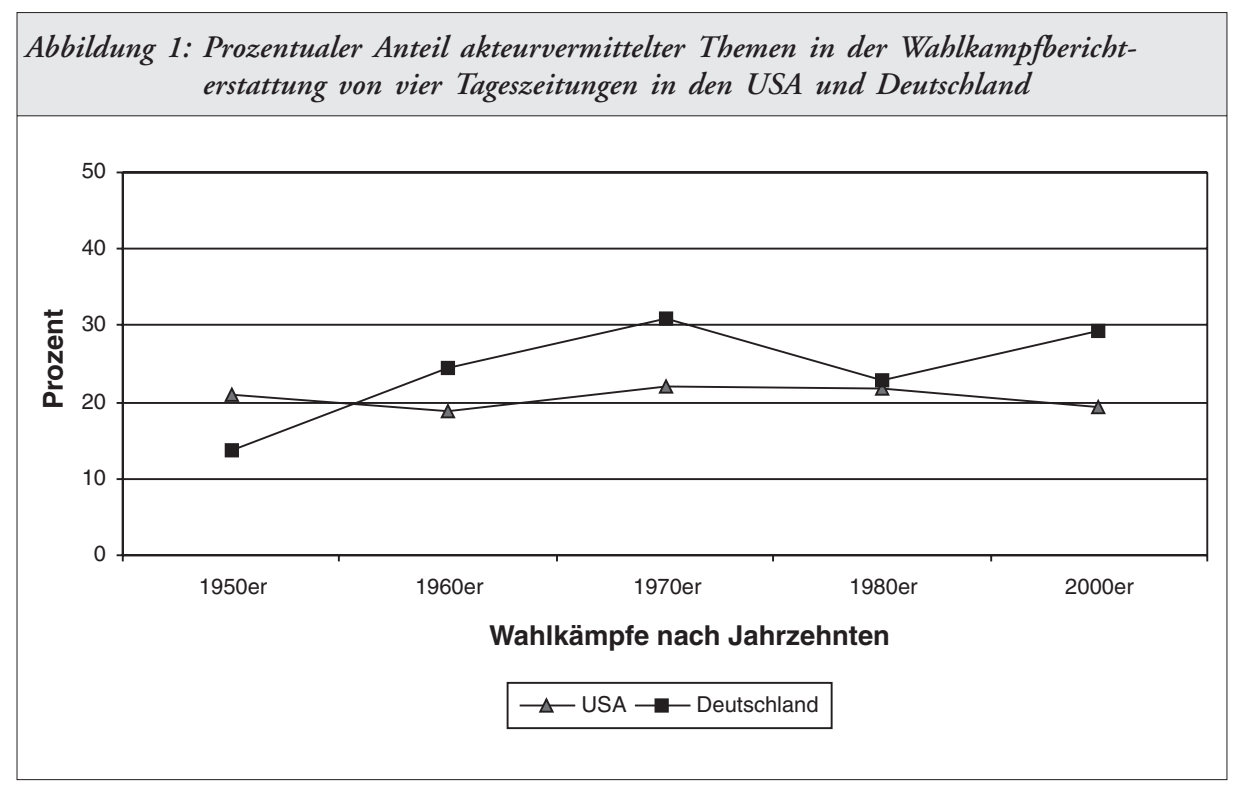

24 Frankfurter Allgemeine Zeitung vom 9. September 2002, Artikel über das zweite TV-Duell zwischen Gerhard Schröder und Edmund Stoiber. 
sich auch in der Berichterstattung wider: Das Spektrum erweitert sich durch die Kabinettsmitglieder und Parteien. Dabei bezieht sich die Aufmerksamkeit nicht nur auf die Parteiführung, sondern auch auf einzelne Parteimitglieder. In den USA ist die Bandbreite der politischen Akteure eingeschränkter. Durch die Konzentration auf die Präsidentschaftskandidaten und die schwache Stellung der Parteien gibt es in der US-amerikanischen Wahlkampfberichterstattung weniger politische Akteure als in Deutschland, mittels derer Themen transportiert werden können. Hier schlägt der Systemtypus „präsidentielle Demokratie" zu Buche. Der amerikanische Präsident allein hat die entscheidenden exekutiven Handlungsbefugnisse inne, ihm stehen keine Minister mit eigenen Einflussbereichen zur Seite.

\subsection{Deutschland}

Die Forschungsliteratur diagnostiziert für deutsche Wahlkämpfe eine Zunahme von Dethematisierung ${ }^{25}$. Dabei wird eine wachsende Konzentration auf Personen und deren apolitische Eigenschaften attestiert, welche die Vermittlung von politischen Sachthemen immer mehr verdrängt. Susan E. Scarrow ${ }^{26}$ spricht gar von einem „deutschen Trend“, bei dem Personen und nicht politische Inhalte in den Mittelpunkt des Wahlkampfes gestellt werden. „Köpfe statt Themen“ lautet der Vorwurf und zugleich die Klage, dass Personen immer mehr als Themensurrogat dienen und die sachpolitische Diskussion während Wahlkämpfen immer mehr ins Abseits drängen. Für die empirische Analyse der Wahlkampfberichterstattung in Deutschland stellt sich offensichtlich die Frage, ob diese Feststellung zutrifft und inwieweit spezifische institutionelle Faktoren hier Einfluss nehmen.

Das politische System Deutschlands zeichnet sich vor allem durch die Schlüsselposition der Parteien aus ${ }^{27}$. Die zentralen politischen Akteure in der Berichterstattung über die untersuchten Bundestagswahlkämpfe sind die Gruppe der Spitzenpolitiker und die Kanzlerkandidaten. Diese vermittelten in allen untersuchten Bundestagswahlkämpfen die meisten Themen. Auffallend ist, dass die Kanzlerkandidaten bis einschließlich der sechziger Jahre die bedeutendsten politischen Akteure im Wahlkampf waren, ab den siebziger Jahren übernahmen die Spitzenpolitiker diese Position (vgl. Abbildung 2). In den sechziger Jahren wurden noch 34 Prozent der Themen in Verbindung mit Spitzenkandidaten vermittelt, in den siebziger Jahren waren es mit 19 Prozent erheblich weniger: Die Kanzlerkandidaten verloren eindeutig an Präsenz. Nicht sie sind es also, sondern die Spitzenpolitiker, denen immer mehr mediale Aufmerksamkeit geschenkt wird. Hier bildet sich für Deutschland vor allem die starke Position einzelner Minister ab. Das Ressortprinzip und das Einflusspotential, das Ministern zu Gebote steht, zeigen sich auch in der Medienberichterstattung. So tauchen die Ressortchefs zugleich als „agenda setter“ und Ex-

25 Vgl. etwa Christina Holtz-Bacha, 2000, a.a.O.; Fritz Plasser, a.a.O.

26 Susan E. Scarrow, Der Rückgang von Parteibindungen aus der Sicht der deutschen Parteien: Chance oder Gefahr?, in: Peter Mair / Wolfgang C. Müller / Fritz Plasser (Hrsg.), Parteien auf komplexen Wählermärkten. Reaktionsstrategien politischer Parteien in Westeuropa, Wien 1999, S. $71-102$.

27 Vgl. Wichard Woyke, Stichwort Wahlen. Wähler - Parteien - Wahlverfahren, Bonn 1994, S. 79 . 


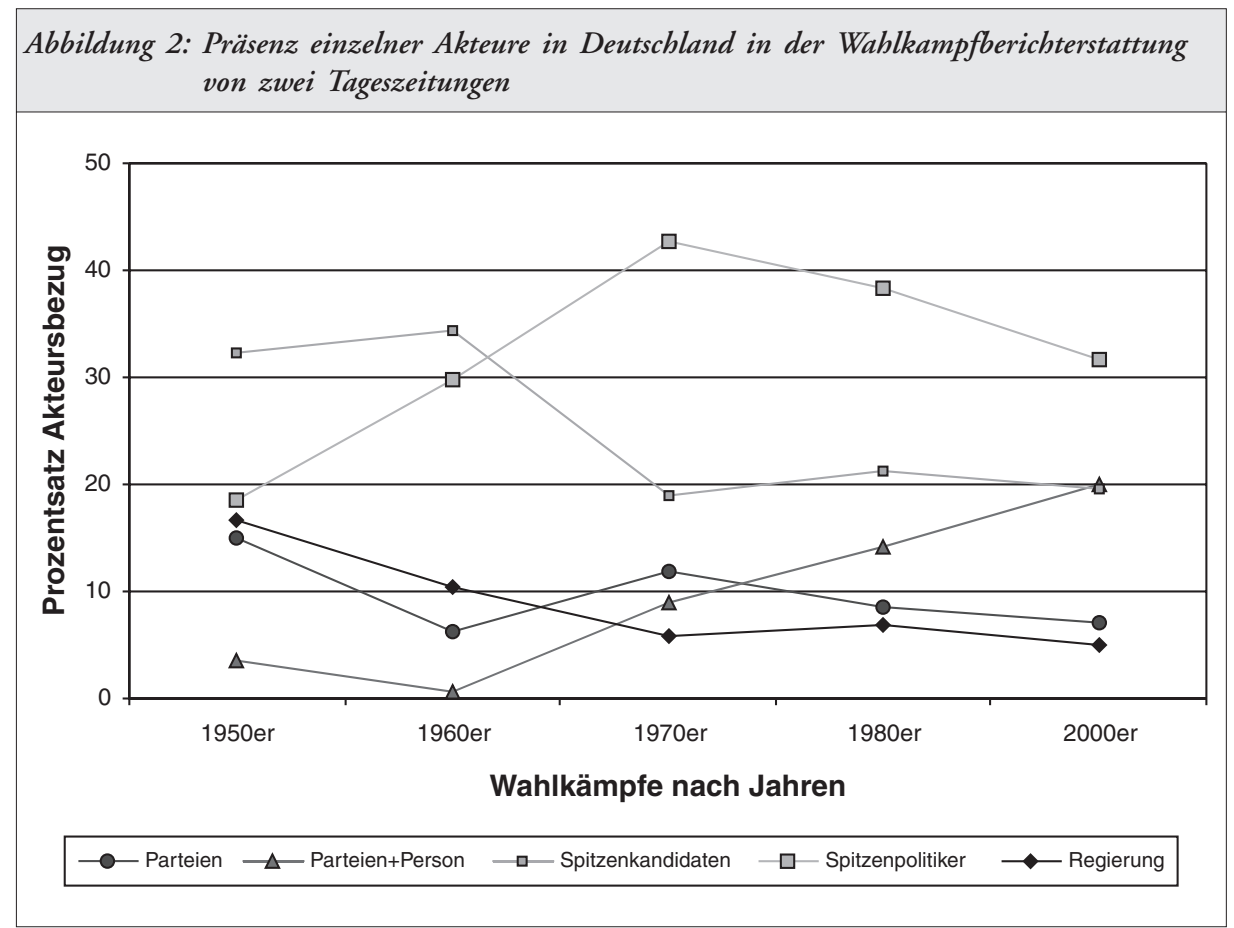

perten für ihren Politikbereich in der Wahlkampfberichterstattung auf und dominieren auf diese Art und Weise die Themenvermittlung.

Sowohl die Parteien als auch die Regierung zeigen sich bei der Themenvermittlung insgesamt schwach. Für beide Akteure lässt sich seit den fünfziger Jahren ein Abwärtstrend feststellen. Trotz der starken Position der Parteien in Deutschland rangieren diese mit Ausnahme der siebziger Jahre stets auf dem vorletzten Platz. Der Anteil an Themen, der in Verbindung mit ihnen vermittelt wurde, ist in den fünfziger Jahren mit 15 Prozent am größten und bewegt sich außer in den siebziger Jahren unter der Zehn-ProzentMarke. Deutschland gilt als „parteienstaatliche Demokratie“ ${ }^{28}$, bei der Themenvermittlung innerhalb der Wahlkampfberichterstattung wird es diesem Ruf jedoch nicht gerecht: Hier dominieren eindeutig einzelne Politiker. Dennoch kann man die Parteien nicht vernachlässigen: Der Anteil an Themen, der in Verbindung mit einer Person und ihrer Partei vermittelt wurde, zeigt seit den sechziger Jahren einen starken Aufwärtstrend: In den sechziger Jahren wurden nur knapp zwei Prozent an Themen in Verbindung mit Partei und Person vermittelt, wohingegen es im darauf folgenden Jahrzehnt schon neun Prozent waren. Die Aufmerksamkeit für Personen ist zwar bei der medialen Berichterstattung stärker, und korporative politische Akteure treten in den Hintergrund; dennoch zeigt sich die tragende Rolle der Parteien in Bezug auf die Personalisierung der Wahlkampfberichterstattung. Es scheint hier eine besondere Art von Personalisierung zu greifen: Perso-

28 Manfred Schmidt, Demokratietheorien. Eine Einführung, 3., überarbeitete und erweiterte Auflage, Opladen 2000, S. $376 \mathrm{ff}$. 
nalisierung, gekoppelt an die Nennung von Parteien. Diese „parteigebundene“ Form tritt in Deutschland mehr und mehr in den Vordergrund.

Dabei handelt es sich nicht nur um die Parteiprominenz, sondern auch um Mitglieder, die keine hervorgehobene Position in der Parteiorganisation oder der Regierung bekleiden. Diese Tatsache kann mit dem Strategiewandel beziehungsweise der Anpassung der Parteien an das Mediensystem erklärt werden. Der Transport von Botschaften mittels Personen stellt eine Strategie dar, mit der die Aufmerksamkeit der Medien gewährleistet ist. Dabei konzentriert sich die Personalisierung nicht mehr nur auf einzelne Spitzenpolitiker innerhalb der Partei, sondern weitet sich auf den gesamten Parteiapparat aus.

Im Zuge des expandierenden Mediensektors, der für die Vermittlung von politischen Botschaften immer mehr an Bedeutung gewinnt, setzt sich in Deutschland eine neuartige Personalisierung der Politik in der Wahlkampfberichterstattung durch. Konzentrierte sich die Personalisierung in den fünfziger und sechziger Jahren noch auf einen kleinen Kreis politischer Akteure, das heißt auf die Spitzenkandidaten und Spitzenpolitiker, so tauchen seit den siebziger Jahren zunehmend Politiker auf der Medienagenda auf, die keine herausragende Bedeutung im politischen System innehaben. ${ }^{29}$ Die Personalisierung nimmt in Deutschland insgesamt zwar nicht zu, sie weitet sich jedoch in Bezug auf die Anzahl der politischen Akteure aus, die sie einbezieht.

\subsection{USA}

In den USA wird die Personalisierung der Politik im Gegensatz zu Deutschland vom politischen System begünstigt. Bei Präsidentschaftswahlen wird die Exekutive direkt gewählt, und die Parteien haben dabei eine eher schwache Stellung, denn sie dienen den Spitzenkandidaten lediglich als Plattform und haben eher eine „Zuarbeiterfunktion“ im Gegensatz zu Deutschland, wo ein Wahlkampf von Partei mit Kandidat geführt wird und die Parteien wesentliche Inhalte und Richtlinien für den Wahlkampf vorgeben ${ }^{30}$.

Wie in Deutschland auch sind in den USA die Spitzenkandidaten und die Gruppe der Spitzenpolitiker die politischen Akteure, die in der Wahlkampfberichterstattung der untersuchten Wahlkämpfe am häufigsten vorkommen. Die Entwicklung ihrer Medienpräsenz verläuft in den USA jedoch gegenläufig zu jener in Deutschland: Die Spitzenkandidaten zeigen mit Ausnahme der fünfziger Jahre die stärkste Präsenz bei der Themenvermittlung, die Spitzenpolitiker rangieren immer auf dem zweiten Platz (vgl. Abbildung 3). Wurden in den fünfziger Jahren noch 36 Prozent der Themen mit Spitzenpolitikern vermittelt, waren es in den sechziger Jahren nur noch 24 Prozent. Dieses Niveau bleibt bis zu den achtziger Jahren konstant, in den 2000er Jahren fällt das Niveau jedoch auf 19 Prozent. Die Präsidentschaftskandidaten sind die stärksten politischen Akteure bei der Themenvermittlung innerhalb der amerikanischen Wahlkampfberichterstattung, ihre zentrale Rolle innerhalb des politischen Systems wird von den Medien abgebildet. Die Konzentration auf sie nimmt dabei nicht linear zu, vielmehr unterliegt die Entwicklung

29 Diese Aussage kann basierend auf einer hier nicht dargestellten Analyse der Kategorie ,andere Politiker" des Kategorienschemas (vgl. Tabelle 1) getroffen werden.

30 Vgl. Christoph Strünck, Agenten oder Agenturen? Amerikanische und deutsche Parteien in vergleichender Perspektive, in: Klaus Kamps (Hrsg.), a.a.O., S.199 - 220; Fritz Plasser, a.a.O. 


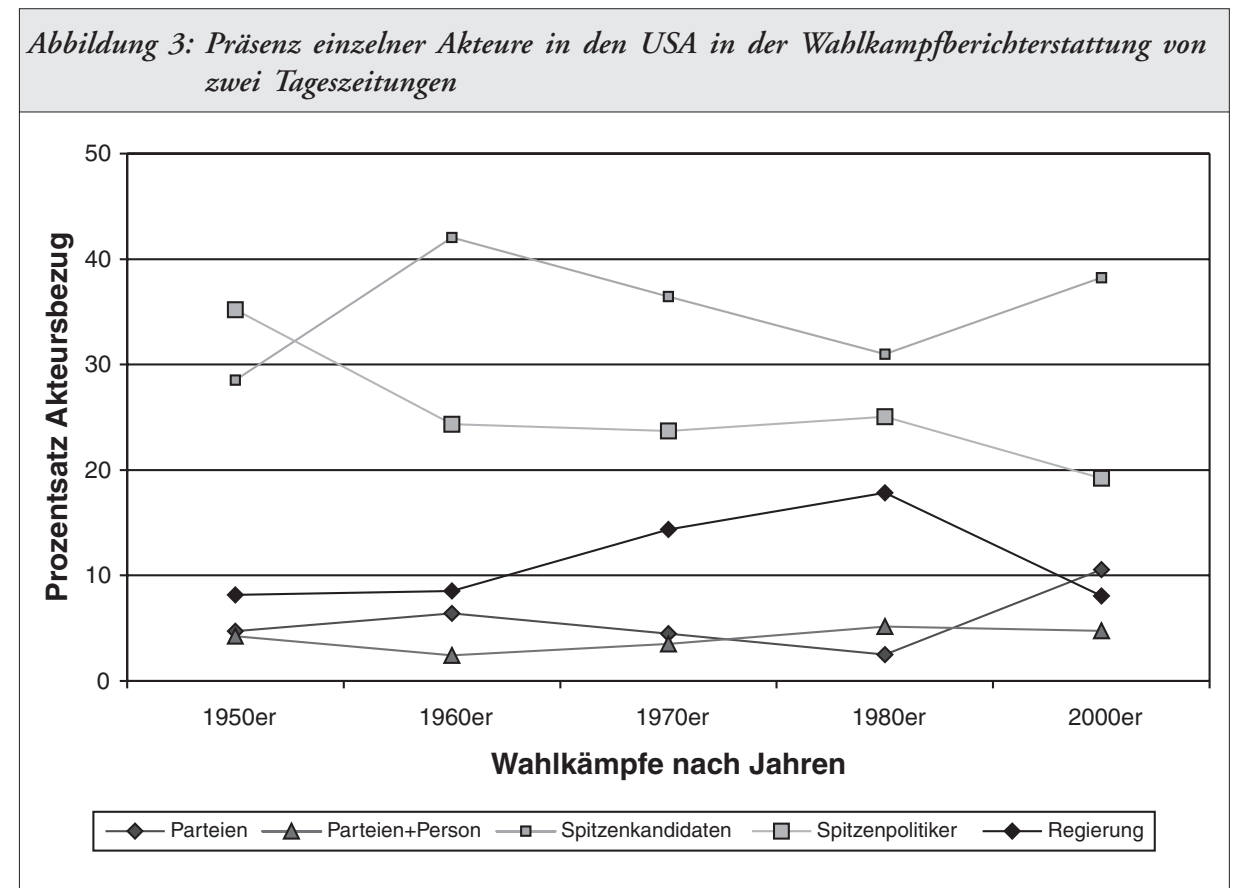

starken Schwankungen: In den sechziger Jahren ist mit 42 Prozent der höchste Anteil an Themen, die in Verbindung mit Spitzenkandidaten vermittelt wurden, zu verzeichnen. Dieses hohe Niveau wird jedoch nicht beibehalten: In den siebziger Jahren lag der Anteil nur noch bei 37 Prozent, und in den achtziger Jahren fiel er auf 31 Prozent ab. Mit 38 Prozent ist in den 2000er Jahren ein Aufwärtstrend zu erkennen, das Niveau der sechziger Jahre wird jedoch nicht erreicht. Unter den in die Untersuchung einbezogenen Präsidentschaftswahlkämpfen ist jener von 2000 der professionalisierteste ${ }^{31}$. So wäre zu erwarten gewesen, dass der Fokus auf die Spitzenkandidaten hier am stärksten zutage tritt. Dies ist jedoch nicht der Fall.

Die Parteien zeigen sich wie in Deutschland eher schwach. Parteien, aber auch - anders als in Deutschland - einzelne Politiker in Verbindung mit ihren Parteien tauchen kaum auf der Medienagenda auf. Die Administration ist der stärkste Akteur nach den Spitzenkandidaten und Spitzenpolitikern. Die generell schwache Stellung der Parteien in den USA schlägt sich also in der Wahlkampfberichterstattung nieder. Als ,party in service“32 konzentrieren sich ihre Aufgaben vor allem auf die Unterstützung des Kandidaten

31 Pippa Norris, Introduction. The Rise of Postmodern Political Communications?, in: dies. (Hrsg.), Politics and the Press. The News Media and Their Influences, London 1997, S. 1 17; David M. Farrell, Modernisierung westeuropäischer Parteien: Ideenkauf auf dem Markt der US-Politik?, in: Matthias Machnig (Hrsg.), Politik - Medien - Wähler. Wahlkampf im Medienzeitalter, Opladen 2002, S. 71 - 96; Andrea Römmele, Parteien und Wahlkämpfe - gestern, heute, morgen, in: ebenda, S. $97-105$.

32 Christoph Strünck, a.a.O., S. 213. 
im Wahlkampf ${ }^{33}$. Die Verbindung zwischen dem Kandidaten und seiner Partei ist von Zweckmäßigkeit geprägt, eine enge ideologische und programmatische Verbindung wie in Deutschland gibt es nicht ${ }^{34}$. Daher tauchen Personen in Verbindung mit Parteien kaum in der Wahlkampfberichterstattung auf und bilden bei der Themenvermittlung auch insgesamt das Schlusslicht.

Die Administration ${ }^{35}$ zeigt vor allem in den siebziger und achtziger Jahren eine starke Präsenz auf der Medienagenda: In den siebziger Jahren wurden 14 Prozent der Themen in Verbindung mit ihr vermittelt, und in den achtziger Jahren stieg der Anteil auf 18 Prozent. Mit dem Bedeutungszuwachs der Administration geht ein Bedeutungsverlust der Spitzenkandidaten einher. Wie lassen sich diese gegenläufigen Trends erklären? Der Präsidentschaftswahlkampf von 1972 wurde stark vom Vietnamkrieg überschattet. Die krisenhafte außenpolitische Lage forderte zahlreiche gewichtige Entscheidungen von der Staatsführung, die so auch von den Medien aufgenommen wurden: Das Vorgehen des Weißen Hauses und der zuständigen Behörden und Departments beim Vietnamkrieg stand im Mittelpunkt des Interesses der Medien; andere politische Akteure verloren an Aufmerksamkeit. Vor allem die Präsidentschaftskandidaten büßten in der Medienberichterstattung an Bedeutung ein, da das Thema Wahlkampf durch den Vietnamkrieg auf der Medienagenda ins Abseits gedrängt wurde. Durch die außenpolitische Ausnahmesituation wurde der Medienfokus von Wahlkampfinhalten und horse race-journalism weggelenkt. Die Präsidentschaftswahl von 1988 zeichnete sich durch einen unspektakulären Wahlkampf aus, was sich auch in der Medienberichterstattung widerspiegelte. In der Wählerschaft herrschte eine allgemeine Zufriedenheit mit dem politischen Status quo und der Sieg von George Bush Senior war absehbar ${ }^{36}$.

33 Vgl. Peter Radunski, Wahlkämpfe, a.a.O., S. 158; Christoph Strünck, a.a.O.

34 Vgl. Barbara Pfetsch / Rüdiger Schmitt-Beck, Amerikanisierung von Wahlkämpfen? Kommunikationsstrategien und Massenmedien im politischen Mobilisierungsprozess, in: Michael Jäckel / Peter Winterhoff-Spurk (Hrsg.), Politik und Medien. Analysen zur Entwicklung der politischen Kommunikation, Berlin 1994, S. 231 - 252, S. 237.

35 Die Administration in den USA setzt sich aus Departments, Dienststellen und Behörden zusammen, deren Chefs direkt vom Präsidenten ernannt werden und seine Politik vertreten und durchsetzen sollen, vgl. hierzu Peter Lösche / Hartmut Wasser, Politisches System der USA. Informationen zur politischen Bildung, Bonn 2004. Im Gegensatz dazu steht die Regierung in Deutschland, die neben dem Bundeskanzler aus den einzelnen Ministern besteht. Hier besitzt der Bundeskanzler zwar die Richtlinienkompetenz, diese wird jedoch durch das Ressortprinzip eingeschränkt: Jeder Minister ist allein verantwortlich für die Entscheidungen, die in seinem Ministerium getroffen werden, der Bundeskanzler kann lediglich die politische Richtung vorgeben. Die Tatsache, dass in Deutschland in der Regel Koalitionsregierungen gebildet werden, schränkt die Richtlinienkompetenz des Kanzlers zusätzlich ein: Das Kabinett enthält Minister, die nicht seiner eigenen Partei angehören, was politische Kompromisse fordert. Vgl. hierzu Wolfgang Rudzio, Das politische System der Bundesrepublik Deutschland, 4. Auflage, Opladen 1996.

36 Vgl. Robert E. Denton / Gary C. Woodward, a.a.O, S. 198 f. 
4. Die Personalisierung der Wahlkampfberichterstattung: ein Negativimage löst sich auf

Zwischen der deutschen und der US-amerikanischen Wahlkampfberichterstattung konnten keine Konvergenzen in Bezug auf die Personalisierung festgestellt werden. Die deutsche Wahlkampfberichterstattung nähert sich im Hinblick auf Personalisierungstendenzen der US-amerikanischen nicht an. In Deutschland richtet sich der Fokus auf andere politische Akteure als in den USA. Insgesamt tauchen auch mehr politische Akteure auf der Medienagenda auf. So machen sich die unterschiedlichen systemischen Rahmenbedingungen der beiden Länder bemerkbar. Institutionelle Filter verhindern eine bloße Übernahme von US-amerikanischen Wahlkampftechniken und fordern eine Anpassung an die länderspezifischen Bedingungen des Wahlkampfes ${ }^{35}$.

Die empirischen Befunde zeigen ferner, dass die Befürchtung, mit der Personalisierung gehe eine Themenarmut einher, entkräftet werden kann. Personen tauchen in Verbindung mit Themen auf der Medienagenda auf, sie dienen als Mittel, den Bürgern politische Themen darzustellen. Der Vorwurf „Köpfe statt Themen“ ist nicht haltbar, „Köpfe und Themen" muss das Motto lauten. Vor allem am Beispiel der Europäischen Union lässt sich ablesen, dass die Informationsvereinfachung, die durch die Personalisierung geleistet wird, heutzutage wichtiger denn je ist. Durch die europäische Mehrebenenverflechtung werden politische Prozesse zunehmend komplexer und undurchschaubarer, und es bedarf eines probaten Mittels, um den Wähler erreichen zu können. Personalisierung kann dazu beitragen und dem Bürger abstrakte politische Prozesse näher bringen.

So kann ein Plädoyer zu Gunsten der Personalisierung entgegen ihres negativen Images in der Fachliteratur gewagt werden: Personalisierung der Politik ist kein bloßes Themensurrogat, sondern stellt ein wirksames Mittel zur Politikvermittlung dar. Politische Kommunikation profitiert von der Personalisierung der Politik.

37 Vgl. shopping model vs. adaption model: Fritz Plasser, a.a.O; Jens Tenscher, Professionalisierung der Politikvermittlung? Politikvermittlungsexperten im Spannungsfeld von Politik und Massenmedien, Wiesbaden 2003. 